\title{
Assessment of deuteron-induced reaction mechanisms at low and medium energies
}

\author{
M. Avrigeanu, a and V. Avrigeanu \\ "Horia Hulubei” National Institute for Physics and Nuclear Engineering, P.O. Box MG-6, 76900 Bucharest, Romania
}

\begin{abstract}
An extended analysis of the nuclear reaction mechanisms involved in the deuterons interaction with ${ }^{63,65} \mathrm{Cu}$ is presented. First, the available elastic-scattering data analysis provided us the optical potential for reaction cross sections calculations. An increased effort has been devoted to the breakup mechanism, both the elastic breakup and the breakup fusion contributions to the different activation cross sections being carefully considered. Next, the direct reaction contributions through the one nucleon stripping reaction cross sections have been calculated. Finally, the pre-equilibrium and compound-nucleus cross-section calculation, corrected for the breakup and stripping decrease of the total reaction cross section, completed the deuteron activation cross-section analysis. The overall agreement between the measured and calculated deuteron activation cross sections proves the correctness of the nuclear mechanisms account.
\end{abstract}

\section{Introduction}

The description of deuteron-nucleus interaction represents an important test for both the quality of reaction mechanism models and evaluation of nuclear data requested especially for fusion reactor technology. The weak binding of the deuteron triggers significant contributions of the breakup channel and enhances a variety of reactions at low bombarding energy which complexity hampers the comprehensive analysis involving large A-range of target nuclei and incident energies domain. Therefore the actual calculations and measured data for deuteron-induced reactions are less extensive and mature than for neutrons, so that improved model calculations and further measurements are needed if the deuteron libraries are to approach the standard of the established neutron ones. These requests motivated a former analysis of the deuteron elastic-scattering and reaction cross sections induced on ${ }^{27} \mathrm{Al}$, at the incident energies from 3 to $60 \mathrm{MeV}[1,2]$, and its extension for the $\mathrm{Cu}$ stable isotopes.

\section{Energy-dependent optical potential parameters}

The simultaneous analysis of the deuteron elastic scattering and induced activation appears essential for a consistent input of nuclear model calculations [2], a prime interest for the optical model potential (OMP) parameters being motivated by their further use in the calculation of deuteron interaction cross sections.

The few measurements of angular distributions of elastic scattered deuterons on the stable isotopes and on natural

\footnotetext{
a e-mail: mavrig@ifin.nipne.ro
}

copper [3], shown Fig. 1, did not allow us to make an extended OMP analysis. On the other hand, while previous OMP analysis on ${ }^{6,7} \mathrm{Li}[4,5]$ and ${ }^{27} \mathrm{Al}[1,2]$ show that no global OMP describes reasonably well the elastic scattering data in the energy range up to $20 \mathrm{MeV}$, slight adjustment of Daehnick et al. [6] parameterization led us to a good description of the data for the copper isotopes (Fig. 1). The comparison of the experimental elastic-scattering angular distributions for ${ }^{63,65, \text { nat }} \mathrm{Cu}$ and the calculated values obtained by using these adjusted OMP parameters (solid curves), the global optical potential [6] (dot-dashed curves), and TALYS [7] code default option (dashed curves) based on the Watanabe folding approach [8] is shown in the left side of the Fig. 1. The measured reaction cross sections for deuterons incident on the ${ }^{63,65} \mathrm{Cu}$ isotopes and natural $\mathrm{Cu}$ [3] are also compared in Fig. 1 with the calculated values obtained by using the same potentials, and the evaluated data within the ACSELAM library [9] (dotted curve). The last two calculated reaction excitation functions underestimate the measured values by at least $20 \%$. Finally, the parameters adjustment is shown in the right lower corner of Fig. 1 and compared with the original values [6]. The elastic-scattering cross section calculations have been performed using the computer code SCAT2 [10].

\section{Deuteron breakup contribution}

For deuteron energies below and around the Coulomb barrier the interaction of deuterons with the target nuclei proceeds largely through direct reaction (DR) processes, while increasing the incident energy other reaction mechanisms like pre-equilibrium emission (PE) or evaporation from the fully equilibrated compound nucleus $(\mathrm{CN})$ also become important. The breakup mechanism responsible for the enhancement of a large variety of reactions is important along 

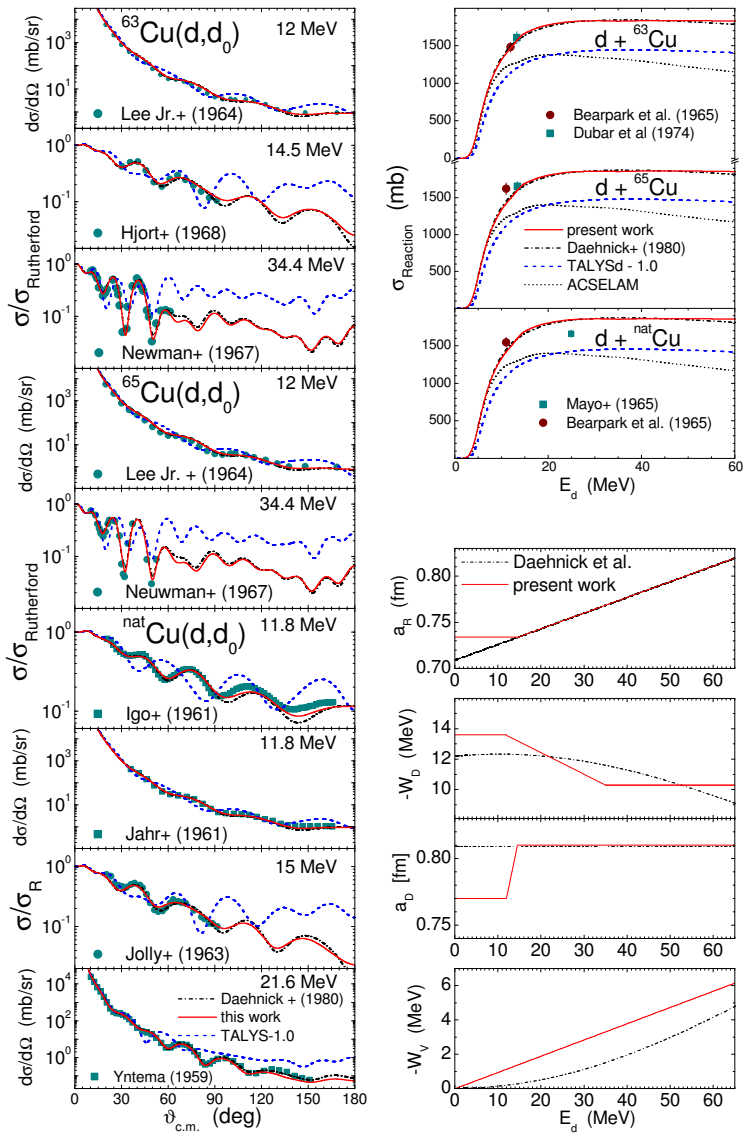

Fig. 1. (Color online) Optical model potential analysis of the deuterons interaction with ${ }^{63,65, \text { nat }} \mathrm{Cu}$ (see details in the text).

the whole incident energy range and its contribution to the reaction cross sections has to be taken explicitly into account [2].

The physical picture of the deuteron breakup in the Coulomb and nuclear fields of the target nucleus considers two distinct chains, namely the elastic breakup (EB) in which the target nucleus remains in its ground state and none of the deuteron constituents interacts with it, and the inelastic breakup or breakup fusion (BF), where one of these deuteron constituents interacts with the target nucleus while the remaining one is detected. An empirical parametrization of the total proton-emission breakup fraction, $\sigma_{B}^{p} / \sigma_{\text {Reaction }}$, and elastic breakup fraction, $\sigma_{E B} / \sigma_{\text {Reaction }}$, have been obtained [1] from the analysis of the experimental systematics [11] of the proton-emission spectra and angular distributions of deuteron-induced reactions on target nuclei from $\mathrm{Al}$ to $\mathrm{Pb}$, at incident energies from 15 to $80 \mathrm{MeV}$. Their dependence with respect to the charge (Z), atomic number of the target nucleus (A), and deuteron incident energy $(\mathrm{E})$ is $[1]$ :

$$
\begin{array}{r}
f_{B}^{(p)}=0.087-0.0066 Z+0.00163 Z A^{1 / 3}+ \\
0.0017 A^{1 / 3} E-0.000002 Z E^{2}, \\
f_{E B}=0.031-0.0028 Z+0.00051 Z A^{1 / 3}+ \\
0.0005 A^{1 / 3} E-0.000001 Z E^{2} .
\end{array}
$$
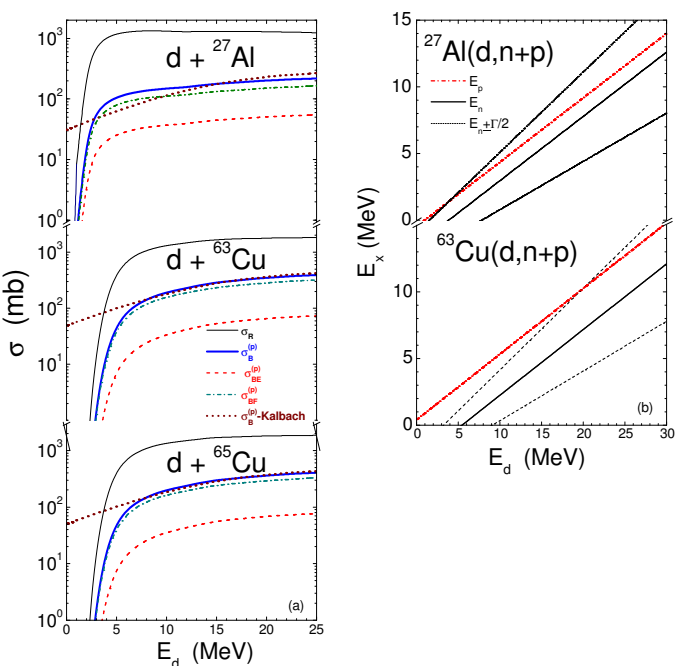

Fig. 2. (Color online) (a) The energy dependence of the total (thick solid), the elastic (dashed), the inelastic (dot-dashed) proton-emission breakup cross sections given by [1] and of the total proton-emission breakup cross sections [12] (dotted) from the deuterons interactions with ${ }^{27} \mathrm{Al}$ and ${ }^{63,65} \mathrm{Cu}$. The corresponding total reaction cross section is shown by thin curve. (b) The centroid $E_{x}$ of assumed Gaussian line shape [12] for deuteron breakup-peak energies of emitted neutrons (solid) and protons (dash-dotted), and the corresponding $E_{n} \pm \Delta$ values (dashed) calculated for deuterons interacting with ${ }^{27} \mathrm{Al}$ and ${ }^{63} \mathrm{Cu}$.

A comparison with total proton- and neutron- emission breakup cross sections parametrization of Kalbach [12]:

$$
\sigma_{B}^{p, n}=K_{d,(p, n)} \frac{\left(A^{1 / 3}+0.8\right)^{2}}{1+\exp \frac{(13-E)}{6}}, \quad K_{d, p}=21, \quad K_{d, n}=18 .
$$

shows that the former parametrization [1] considers equal breakup fractions for proton and neutron emission, but supplementary gives all breakup components, i.e. the total, elastic, and inelastic fraction $f_{B F}^{(p, n)}=f_{B}^{(p, n)}-f_{E B}$. It can be seen in Fig. 2(a) that both parameterizations of Refs. [1, 12] for ${ }^{63,65} \mathrm{Cu}$ target nuclei are very close for deuteron incident energies above $\sim 7 \mathrm{MeV}$, while decreasing the incident energy the total proton-emission breakup cross sections given by Kalbach parametrization [12] remain higher than even the deuteron total reaction cross sections (thin solid curves in Fig. 2(a)).

On the whole, the deuteron total breakup reaction cross section reduces the amount of the total reaction cross section that should be shared among different outgoing channels. On the other hand, the inelastic breakup process, where one of deuteron constituents interacts with the target leading to a secondary composite nucleus, brings contributions to different reaction channels, enhancing mostly the secondarychance emission of particles from the original d-target interaction. Therefore the absorbed neutron following the breakup proton emission contributes to the enhancement of the $(d, p)$ but mainly $(d, 2 p)$ and $(d, p \alpha)$ reaction cross sections. In order to calculate this enhancement, the inelastic breakup cross section, $\sigma_{B F}^{(p)}$, has been multiplied by the corresponding fraction leading to the above-mentioned reactions, e.g. 
$\sigma_{(n, x)} / \sigma_{\text {Reaction }}$, where $\sigma_{\text {Reaction }}$ is the total neutron reaction cross section and $s$ stands for $\gamma, p$, or $\alpha$ outgoing channel [2]. A similar procedure has been followed in order to obtain the contribution to the $(d, 2 n)$ reaction cross section due to the protons which, following the breakup neutron emission, are absorbed in further interactions with the target nucleus.

The inelastic breakup cross section has been expressed as a function of the deuteron incident energy while the Kalbach [12] formula has been used for the centroid energy of the absorbed nucleon from the breakup, responsible for the specific reaction enhancements. The quite large widths of the assumed Gaussian line shape of these peaks as showed in Fig. 2(b) emphasize however the broad approximation of this method. Therefore, the related contributions to the deuteron activation reaction cross sections, provided by the above-mentioned multiplication, have been additionally smoothed by using an average energy width of $3 \mathrm{MeV}$, with the results shown by thin solid curves in Figs. 3(c-f).

The inelastic breakup component still remains difficult to be handled in the reaction calculations by using the available codes. Thus, Kalbach's [12] absolute total neutron and proton breakup cross sections are considered in the TALYS-1.0 code [7] for the first-chance neutron and respectively proton emission spectra while the consideration of a particular contribution following the absorption of one $\mathrm{BF}$ proton or neutron by the target remains an open question.

\section{Single-nucleon stripping}

Apart from the deuteron breakup contributions to (d,p) reactions, the DR one-nucleon striping contribution to the $(\mathrm{d}, \mathrm{p})$ reaction cross sections for the ${ }^{63,65} \mathrm{Cu}$ isotopes (dashdotted curves in Figs. $3(a-b)$ ), have been calculated by means of the code FRESCO [13] based on the CoupledReaction Channels (CRC) method. The post form distorted wave transition amplitude with finite-range interaction has been chosen. The above-mentioned deuteron phenomenological optical model parameter set has been used for the incident channel, while the Koning-Delaroche [14] OMP global parameters have been used for protons interactions with the residual nuclei. The neutron-proton interaction $\mathrm{V}_{n p}$ was assumed to have a Gaussian shape which parameters were determined from the fit of the deuteron binding energy [15]. The transfered neutron bound states were generated in a Woods-Saxon real potential with global reduced radius of $1.25 \mathrm{fm}$ and diffuseness of $0.65 \mathrm{fm}$, while its depth has been adjusted to the nucleon binding energies in the residual nuclei.

The single neutron stripping $(d, p)$ reaction cross section calculations involved transitions to 63 final states of the odd-odd residual nucleus ${ }^{64} \mathrm{Cu}$ and to 52 final states of the odd-odd residual nucleus ${ }^{66} \mathrm{Cu}$ extending up to $\sim 3$ $\mathrm{MeV}$, for which spectroscopic factors exist. These spectroscopic factors were obtained experimentally from proton angular distribution measurements by Park and Daehnick (see Table II of Ref. [16] and Table I of Ref. [17]).

\section{Pre-equilibrium and statistical emission}

The PE and CN cross sections, corrected for the breakup and stripping decrease of the total reaction cross section, have been obtained by using the computer code STAPRE$\mathrm{H}[18]$ and the consistent local parameter set, including the formerly mentioned phenomenological OMPs.

The nuclear level densities were derived on the basis of the back-shifted Fermi gas (BSFG) formula [19], for the excitation energies below the neutron-binding energy, with small adjustments of the parameters $a$ and $\Delta$ obtained by a fit of more recent experimental low-lying discrete levels [20] and $s$-wave nucleon resonance spacings $D_{0}$ [21]. Above the neutron binding we took into account the washing out of shell effects within the approach of Ignatyuk et al. [22] and Junghans et al. [23], and using the method of Koning and Chadwick [24] for fixing the appropriate shell correction energy. A transition range from the BSFG formula description to the higher energy approach has been chosen between the neutron binding energy and the excitation energy of $15 \mathrm{MeV}$, mainly in order to have a smooth connection. On the other hand, the spin distribution has been determined by a variable ratio $I / I_{r}$ of the nuclear moment of inertia to its rigid-body value, between 0.5 for ground states, 0.75 at the neutron binding energy, and 1 around the excitation energy of $15 \mathrm{MeV}$. Concerning the particle-hole state density which for the PE description plays the same role as the nuclear-level density for statistical model calculations, a composite formula [25] was used within the GDH model with no free parameter except for the $\alpha$-particle state density $g_{\alpha}=A / 10.36 \mathrm{MeV}^{-1}[26]$. The initial configuration of 2 excited particles $(p)$ and 1 hole $(h)$, for deuteron-induced reactions $2 p-1 h$ has been used [11].

The measured [3] and the calculated activation cross sections for ${ }^{63,65} \mathrm{Cu}$ (thick curves) as a sum of the inelastic breakup contribution, the DR cross sections provided by the code FRESCO and the PE+CN contributions supplied by STAPRE-H are shown in Fig. 3, together with the global predictions given by EMPIRE [27] (dotted curves) and TALYS [7] (dashed curves) codes. The comparison among the experimental data and the three calculated activation cross sections (thick, dotted and dashed curves) points out the usefulness of an appropriate consideration of the deuteron-breakup and for the $(d, p)$ activation cross sections of the DR contributions too.

\section{Conclusions}

The main aim of this work has been to present an unitary analysis of the nuclear reaction mechanisms responsible for the deuteron interactions with ${ }^{63,65} \mathrm{Cu}$ target nuclei. The agreement between the measured data and model calculations proves the correctness of nuclear mechanism description taken into account for the deuteron-nucleus interaction. Also, the simultaneous analysis of the deuteron elastic scattering and induced activation appears essential for a consistent input into the nuclear reaction model calculations. 

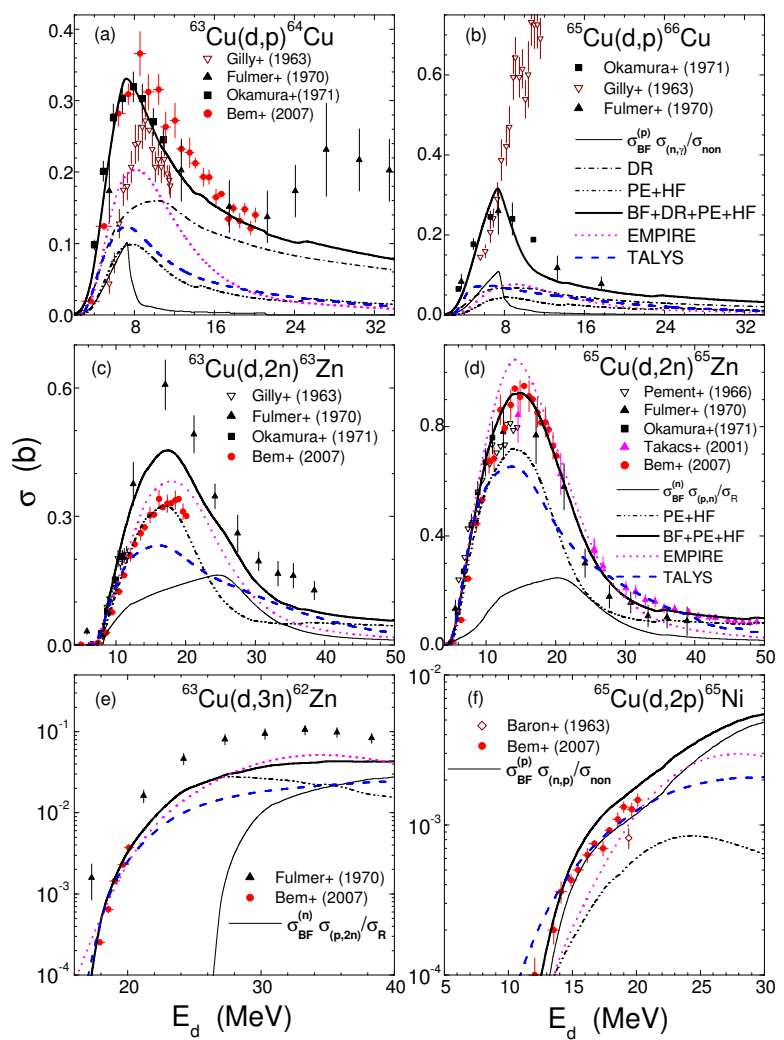

Fig. 3. (Color online) Comparison of measured [3] and calculated reaction cross sections provided by the EMPIRE(dotted) and TALYS (dashed) codes, and present analysis (thick solid) taking into account the deuteron inelastic breakup (thin solid), the DR (dash-dotted), and the PE+CN (dash-dott-dotted) mechanisms contributions to the deuteron interaction with ${ }^{63,65} \mathrm{Cu}$.

Finally the comparison of the present calculations with predictions of the EMPIRE and TALYS codes stress out the importance of the appropriate consideration of the deuteron breakup mechanism contribution to the activation crosssection calculations.

This work was supported by the CNCSIS-Bucharest Contract No. PN-II-ID-PCE-2007-885-149.

\section{References}

1. M. Avrigeanu, W. von Oertzen, R. A. Forrest, A.C. Obreja, F. L. Roman, V. Avrigeanu, Fusion Eng. Design 84 (2009) 418

2. P. Bém, E. Šimečková, M. Honusek, U. Fischer, S. P. Simakov, R. A. Forrest, M. Avrigeanu, A. C. Obreja, F. L. Roman, V. Avrigeanu, Phys. Rev. C 79 (2009) 044610

3. Experimental Nuclear Reaction Data (EXFOR); www-nds.iaea.or.at/exfor

4. M. Avrigeanu, W. von Oertzen, U. Fischer, V. Avrigeanu, Nucl. Phys. A 759 (2005) 327

5. M. Avrigeanu, W. von Oertzen, H. Leeb, F.L. Roman, V. Avrigeanu, in Proc. 11th Int. Conf. on Nuclear Reaction Mechanisms, 12-16 June 2006, Varenna, Italy, edited by E. Gadioli (Ricerca Scientifica ed Educazione Permanente, Milano, 2006), p. 193

6. W. W. Daehnick, J. D. Childs, Z. Vrcelj, Phys. Rev. C 21 (1980) 2253

7. A.J. Koning, S. Hilaire, M.C. Duijvestijn, in Proc. Int. Conf. on Nuclear Data for Science and Technology, Nice, 2007, edited by O. Bersillon, F. Gunsing, E. Bauge, R. Jacqmin, S. Leray (EDP Sciences, Paris, 2008), p. 211

8. S. Watanabe, Nucl. Phys. 8 (1958) 484

9. N. Yamano, Table of Isotope Production Cross Sections (ACSELAM Library), http://wwwndc.jaea.go.jp/ftpnd/sae/acl.html

10. O. Bersillon, Code SCAT2, Note CEA-N-2227, 1992

11. M.G. Mustafa, T. Tamura, T. Udagawa, Phys. Rev. C 35 (1987) 2077 G. Baur, F. Rosel, D. Trautmann, R. Shyam, Phys. Rep. 5 (1984) 333 N. Matsuoka et al., Nucl. Phys. A 391 (1982) 357; J. Kleinfeller et al., Nucl. Phys. A 370 (1981) 205; J.R. Wu, C.C. Chang, H.D. Holmgren, Phys. Rev. C 19 (1979) 370 J. Pampus et al., Nucl. Phys. A 311 (1978) 141

12. C. Kalbach Walker, TUNL Progress Report XLII (2002-2003) p. 80-83, www.tunl.duke.edu/publications/tunlprogress/2003/

13. I.J. Thompson, Comput. Phys. Rep. 7 (1988) 167; version FRES 2.3, May 2007

14. A.J. Koning, J.P. Delaroche, Nucl. Phys. A 713 (2003) 231

15. M. Kamimura et al., Prog. Theor. Phys. Suppl. No. 89 (1986) 1

16. Y.S. Park, W.W. Daehnick, Phys. Rev. 181 (1969) 1082

17. W.W. Daehnick, Y.S. Park, Phys. Rev. 180 (1969) 1062

18. M. Avrigeanu, V. Avrigeanu, IPNE Report NP-861995, Bucharest, 1995, and Refs. therein; News NEA Data Bank 17 (1995) 22

19. H. Vonach, M. Uhl, B. Strohmaier, B. W. Smith, E. G. Bilpuch, G.E. Mitchell, Phys. Rev. C 38 (1988) 2541

20. Evaluated Nuclear Structure Data File (ENSDF); http://www.nndc.bnl.gov/ensdf/index.jsp

21. IAEA-CRP Reference Input Parameter Library (RIPL-2), http://www-nds.iaea.or.at

22. A. V. Ignatyuk, G. N. Smirenkin, A. S. Tishin, Yad. Fiz. 21 (1975) 485; Sov. J. Nucl. Phys. 21 (1976) 255

23. A. R. Junghans, M. de Jong, H.-G. Clerc, A. V. Ignatyuk, G. A. Kudyaev, K.-H. Schmidt, Nucl. Phys. A629 (1998) 635

24. A. J. Koning, M. B. Chadwick, Phys. Rev. C 56 (1997) 970

25. M. Avrigeanu, V. Avrigeanu, Comp. Phys. Comm. 112 (1998) 191 ; A. Harangozo, I. Stetcu, M. Avrigeanu, V. Avrigeanu, Phys. Rev. C 58 (1998) 295

26. E. Gadioli, E. Gadioli-Erba, Z. Phys. A 299 (1981) 1

27. M. Herman, P. Oblozinsky, R. Capote, M. Sin, A. Trkov, A. Ventura, V. Zerkin, in Proc. Int. Conf. on Nuclear Data for Science and Technology, Santa Fe, 2004, edited by R.C. Haight et al. (American Institute of Physics, New York, 2005), p. 1184; EMPIRE-II v.2.19, http://www-nds.iaea.org/empire/ 